\title{
A COMPUTATIONAL TOOL FOR BAYESIAN NETWORKS ENHANCED WITH RELIABILITY METHODS
}

\author{
Silvia Tolo ${ }^{1}$, Edoardo Patelli ${ }^{1}$, Michael Beer ${ }^{1}$, Zhan Kang $^{2}$ \\ ${ }^{1}$ Institute for Risk and Uncertainty \\ University of Liverpool, The Quadrangle, Liverpool, UK \\ e-mail: s.tolo@liverpool.ac.uk \\ ${ }^{2}$ State Key Laboratory of Structural Analysis for Industrial Equipment \\ Dalian University of Technology, Dalian 116024, China
}

Keywords: Bayesian Network, Structural Reliability, Convex Models, Intervals, OpenCossan, Uncertainty.

\begin{abstract}
A computational framework for the reduction and computation of Bayesian Networks enhanced with structural reliability methods is presented. During the last decades, the inner flexibility of the Bayesian Network method, its intuitive graphical structure and the strong mathematical background have attracted increasing interest in a large variety of applications involving joint probability of complex events and dependencies. Furthermore, the fast growing availability of computational power on the one side and the implementation of robust inference algorithms on the other, have additionally promoted the success of this method. Inference in Bayesian Networks is limited to only discrete variables (with the only exception of Gaussian distributions) in case of exact algorithms, whereas approximate approach allows to handle continuous distributions but can either result computationally inefficient or have unknown rates of convergence.

This work provides a valid alternative to the traditional approach without renouncing to the reliability and robustness of exact inference computation. The methodology adopted is based on the combination of Bayesian Networks with structural reliability methods and allows to integrate random and interval variables within the Bayesian Network framework in the so called Enhanced Bayesian Networks. In the following, the computational algorithms developed are described and a simple structural application is proposed in order to fully show the capability of the tool developed.
\end{abstract}




\section{INTRODUCTION}

The fast technological growth that has characterized the last century has progressively provided more efficient and advanced instruments for everyday life as well as for industrial and scientific applications. This progress goes along with an ever increasing grade of complexity which concerns the engineering field on any level, from industrial processes and technological installations to devices of common and daily use. To adequately predict the behaviour of these systems, optimize their performance, estimate their reliability and evaluate the risks they are subject to, are challenging tasks. Hence, it is of primary importance to provide tools suitable for the accurate representation not only of systems but also of data available.

The first of these aspects focuses mainly on the representation, as precise as possible, of the network of dependencies and interactions among the parts which determine the correct functioning of the overall system. The success of Bayesian Networks (BNs) is linked to their capability to satisfy this requirement. Thanks to the robustness of Bayesian probability, they provide an efficient factorization of joint probability distributions exploiting information about the conditional dependencies existing among the variables involved. In addition, their intuitive graphical framework has consolidated their attractiveness in quite different fields of science and engineering, from artificial intelligence to medical and economic areas [21].

On the other side, representing as faithfully as possible the information available is a crucial aspect of modelling. In fact, including in the modelling the lack of knowledge (i.e. epistemic uncertainty) generally leads to a drastic improvement in the accuracy and reliability of the results even if at the cost of a higher computational effort. This approach results in larger robustness, not only avoiding bias and assumptions which can easily lead to misleading results but also allowing to reduce the cost associated with more meticulous measurements. Examples can be found in the structural reliability area which, as the related scientific literature shows, provides a fertile breeding ground for this kind of approach such as imprecise probabilities theory [1].

The core of this study is to provide an efficient computational tool which combines two solid and well consolidated methods (e.g. Bayesian Networks and Structural Reliability methods considering epistemic uncertainty), fully exploiting the advantages of both.

In the following, the theoretical background and the methodology adopted are firstly introduced. The main features of the computational framework developed are then described. Finally, a simple application is presented in order to provide a basis for discussion towards the advantages and limitations of the implementation.

\section{THEORETICAL BACKGROUND}

This section is dedicated to the overall theoretical background of the methodology adopted. A brief introduction to BNs and structural reliability methods is provided. Finally, the methodology adopted is described.

\subsection{Bayesian Networks}

BNs, also known as belief networks, are statistical models based on the use of directed acyclic graphs for the representation of probability distributions. They provide the factorization of the joint probability distribution associated with an event of interest exploiting information about the conditional dependencies existing among the variables. This approach relies on a double nature graphically represented by the structure of the network itself, to which quantitative values are associated throughout the introduction of conditional probability distributions. The structure of a BN consists of a variable number of nodes, each of which represents a ran- 
dom variable of the problem modelled. The variables should be interpreted in Bayesian terms or rather can have different origins: for instance, they may be observable quantities, unknown parameters or even mere hypotheses.

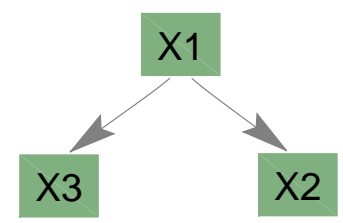

Figure 1: Example of an elementary BN

The nodes of a BN are connected to each other by edges (commonly represented as arrows) expressing informal or causal dependencies existing among the variables. Only nodes among which exists some sort of dependency are linked, whilst those that are not joined refer to variables that are conditionally independent of each other. The edges are characterized by precise directions coherent with the causal relationship of the variables connected. With regards to the BN introduced in Fig. 1, the node $X_{1}$ is called the parent of $X_{2}$ and $X_{3}$, which are also referred to as its children. Nodes that have no parents are defined as the roots of the network. Generally, on the basis of the Bayes' theorem, the joint probability modelled by any BN with nodes $X_{1}, X_{2}, \ldots, X_{n}$ can be expressed as:

$$
P\left(x_{1} \ldots x_{n}\right)=\prod_{i} P\left(x_{i} \mid p_{i}\right)
$$

where $p_{i}$ refers to the outcomes assumed by the parents of the node $X_{i}$, whose state is represented by $x_{i}$. Then, the joint probability associated with the BN of Fig. 1 is:

$$
P\left(x_{1}, x_{2}, x_{3}\right)=P\left(x_{1}\right) P\left(x_{2} \mid x_{1}\right) P\left(x_{3} \mid x_{1}\right)
$$

In a BN each node is conditionally independent of its non-descendants given its parent variables, satisfying the local Markov property [17]. The strength of the dependencies associated with each cluster of parent-child nodes is represented by the conditional probabilities mentioned. These can be of different nature according to the structure of the variables concerned. BNs also allow the updating of the marginal probabilities of the variables involved on the basis of new information that might become available. This way, introducing evidence in the model, it is also possible to analyse "what if" scenarios, as well as the propagation of the information in the direction of interest. Different software packages have been developed which allow the adoptions of several algorithms, both exact and approximate, for the computation of inference in BNs. Please see Ref. [11] for a review of the software packages available. A complete overview of Bayesian networks is provided by Pearl and Russell [15].

\subsection{Bayesian Networks Enhanced with Structural Reliability Methods}

As mentioned in Section 2.1, two options are available for BNs inference computation, namely approximate and exact algorithms. The choice of one or the other approach entails advantages and drawbacks: the approximate approach allows to perform the inference on continuous nodes using simulation techniques (e.g. Markov chain Monte Carlo methods) but can 
result either computationally inefficient or have unknown rates of convergence. On the other hand, exact inference algorithms (e.g. junction tree algorithm) are robust and well-established in scientific literature but, due to computational costs, restrict the use of probability distributions to the discrete field with the only exception of Gaussian distributions [13]. In most cases, this implies the necessity to discretize continuous random variables, impoverishing the quality of the information [19]. The integration of the BN approach with system reliability methods allows to avoid this practise, benefiting of capabilities of exact inference algorithms and overcoming their drawbacks. The resulting strategy is commonly known as Enhanced Bayesian Networks (EBNs) [18].

The role of system reliability methods is to reduce the initial EBN (including discrete as well as continuous variables) to a traditional $\mathrm{BN}$ on which it is possible to compute exact inference. More in detail, each node child of at least one continuous node has to be defined as a domain (or a set of domains) in the outcome space of its parents. This way, the use of system reliability methods, not only allows to compute conditional probability values (as in traditional BNs) starting from continuous variables but also erases the dependency of the computed node from its non-discrete parents. Hence, the links among continuous and discrete nodes can be completely removed, finally allowing the elimination of all not-discrete nodes. The joint probability
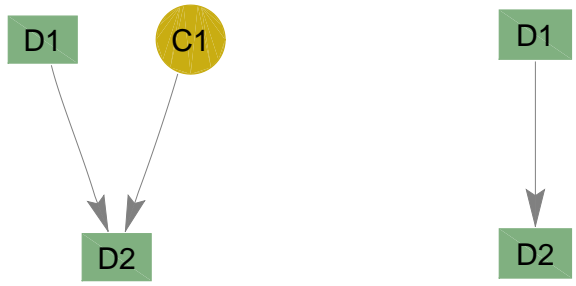

Figure 2: Example of an elementary EBN on the left hand side and its reduced network on the right, where $\mathrm{C} 1$ refers to a continuous whereas D1 and D2 to a discrete node

associated to the reduced network in Fig. 2 can be represented according to the integral of Eq. 1 and then computed as:

$$
P(D 1, D 2)=\int_{C_{1}} p\left(D_{1}\right) p\left(D_{2} \mid D_{1}, C_{1}\right) f\left(C_{1}\right) d C_{1}
$$

where $p\left(D_{1}\right), p\left(D_{2} \mid D_{1}, C_{1}\right)$ are the probability values associated to the discrete nodes $D_{1}, D_{2}$ whilst $f\left(C_{1}\right)$ is the probability density function associated to the continuous node $C_{1}$. Considering the Markov condition, hence the independence of the node $D_{1}$ from the continuous node $C_{1}$, the solution of the integral in Eq. 3 is reduced to:

$$
P(D 1 \mid D 2)=\int_{C_{1}} p\left(D_{2} \mid D_{1}, C_{1}\right) f\left(C_{1}\right) d C_{1}
$$

Bearing in mind the initial hypothesis, any state $d_{2}$ of the node $D_{2}$ can be expressed as domain in the outcome space of the nodes $C_{1}$ and $D_{2}$. The integral can be then expressed as:

$$
P(D 1 \mid D 2)=\int_{\Omega_{D 2, d 1}^{d 2}} f\left(C_{1}\right) d C_{1}
$$


where $\Omega_{D 2, d 1}^{d 2}$ defines the domain of the event $D_{2}=d_{2}$ in the space of $C_{1}$ given the event $D_{1}=d_{1}$. The integral in Eq.5 appears in the form common to structural reliability problems and can be easily solved using structural reliability methods, as shown in the following section. Since the reduction procedure implies the elimination of all continuous nodes, it is clear that, in order to keep in the reduced network variables initially defined as continuous, it is necessary to discretize them. In particular, this results necessary when such variables receive evidence or the computation of their marginal probabilities is required. In light of this, discretization still plays a major role in the modelling and computation of EBN.

\subsubsection{Structural Reliability Methods}

In the field of structural reliability, the domain bounding failure events is generally described by the so-called limited state functions $G(\mathbf{x})$, which represent the failure modes of the system under study. In light of this, considering an event $\mathrm{F}$ defined as a domain in the outcome space of $m$ stochastic variables $\mathbf{x}=\left(x_{1}, x_{2}, \ldots, x_{m}\right)$, the $\mathrm{m}$-dimensional space can be divided in a safe region, represented by the domain $\Omega_{s}=\{\mathbf{x}: G(\mathbf{x})>0\}$, whilst the failure domain can be expressed as $\Omega_{f}=\{\mathbf{x}: G(\mathbf{x}) \leq 0\}$. Hence, the probability of occurrence of the event $\mathrm{F}$ can be quantified solving the integral of the form:

$$
P(f)=\int_{\mathbf{x} \in \Omega_{f}(\mathbf{x})} f(\mathbf{x}) d \mathbf{x}
$$

where $f(\mathbf{x})$ is the joint probability density function associated to the $m$ stochastic variables $\mathbf{x}$. Various methods for the solution of the integral in Eq. 6 are available such numerical integration techniques, Monte Carlo simulations [5] and asymptotic Laplace expansions [16]. Other common solutions (e.g. First-Order and Second-Order Reliability Methods) rely instead on the transformation of the reliability problem previously described into an optimization one [6]. In this case, the random variables in the vector $\mathbf{x}$ are mapped into independent standard normal variables and the minimum distance $\beta$ between the limit state and the origin of the transformed space identified (i.e. the so called reliability index). The probability of failure is then computed on the basis of the assumption introduced. For example, in case of First Order Reliability Method (FORM) a linear approximation of the limit-state function is adopted leading to $P_{f}=\Phi(-\beta)$.

A more generalized approach which aims to take into account epistemic uncertainty has to allow for uncertainty in both the structural parameters and those of the probabilistic models. As for the probabilistic structural reliability, different methods are available, although the related literature is by far more limited than the previous case. One of the most common approach consists of treating epistemic uncertainty using set of descriptor values, such as in the case of intervals [10], convex models [2], random sets [20] and fuzzy sets [22].

In this study only the epistemic uncertainty affecting the structural parameters of the model has been taken into account. On the contrary, no imprecision is considered for the hyperparameters of the random variables which hence are considered as precise probability distributions. The implementation has been restricted to the adoption of two methods.

The first, developed by Luo et. al [8], is based on a nested minimization problem. Thanks to the combination of uncertain parameters in the form of interval variables $(\mathbf{v})$ represented by convex models [7] and precise random variables (u), the limit state can be expressed as a function of both the sets $(G(\mathbf{u}, \mathbf{v})=0)$ and results in a cluster of limit-state surfaces in the standard normal space. The method allows to seek the worst-case combination of interval 
variables value, identifying the limit state surface on which the most probable failure point lies. The inverse of the normal cumulative distribution function of the distance between the identified point and the origin of the standard normal space is assumed to be the upper bound of the failure probability. This approach can be considered a more general case of the probabilistic method FORM: in fact, when the epistemic uncertainty drops the intervals representing the uncertain variables collapse into single values leading back to the traditional FORM procedure.

The second method adopted has been developed by De Angelis et al. [3] and provides the estimation of set-valued failure probabilities. The approach consists of coupling advanced Monte Carlo methods (i.e. Adaptive Line Sampling) with optimization methods in order to estimate the lower and upper bounds of the failure probability. Moreover, the method allows for both imprecise probability distribution functions (i.e. credal sets) and sets of bounded variables.

\section{NUMERICAL IMPLEMENTATION}

The aim of this research is to enrich and implement computationally the existent methodology described in section 2.2. To achieve this goal, structural reliability methods able to include non probabilistic variables such intervals have been integrated into the BN approach. This way not only continuous but also interval variables can be considered and included in the user's models, adding further novelty to the computational tool developed. The EBN methodology previously depicted has been extended and implemented in the general purpose software OpenCossan in an object oriented fashion, ensuring programming flexibility and avoiding code duplication. OpenCossan is a collections of methods and tools under continuous development, coded exploiting the object-oriented Matlab programming environment. It allows to define specialized solution sequences including any reliability method. Furthermore, thanks to the strong flexibility, new reliability methods or optimization algorithm can be easily added. The computational framework is organized in classes, i.e. data structures consisting of data fields and methods together with their interactions and interfaces [14]. Objects (i.e. instances of classes) can be then easily aggregated, forming more complex objects and being processed according to the related methods in order to obtain the output of interest. The numerical implementation developed in this study consists mainly of two classes: the first of these, Node, provides the basic input of the graphical model; the combination of more object Node allows the construction of EnhancedBayesianNetwork objects, defined by their namesake class. These two classes together provide the graphical and numerical implementation of the Enhanced Bayesian Network model. Moreover, their interaction with the reliability methods available in the OpenCossan framework allows the reduction of the initial network to a $\mathrm{BN}$, according to the methodology previously discussed. Finally, the computation of inference in the network is carried out thanks to the interaction of the tool with the Bayes' Toolbox for Matlab [12]. Figure 3 depict the main structure of the computational tool implemented.

This section aims to give an overall description of the computational tool presented. In the following, the two main classes directly related to the implementation of graphical models are described.

\subsection{Node Class}

The basic objects defined by the user in the network design phase belong to the class Node. The main properties associated to an object Node are shown in Table 1.

The nature of the Conditional Probability Distribution (CPD) depends on the node type: in case of discrete nodes it results in a traditional Conditional Probability Table (CPT), in case 


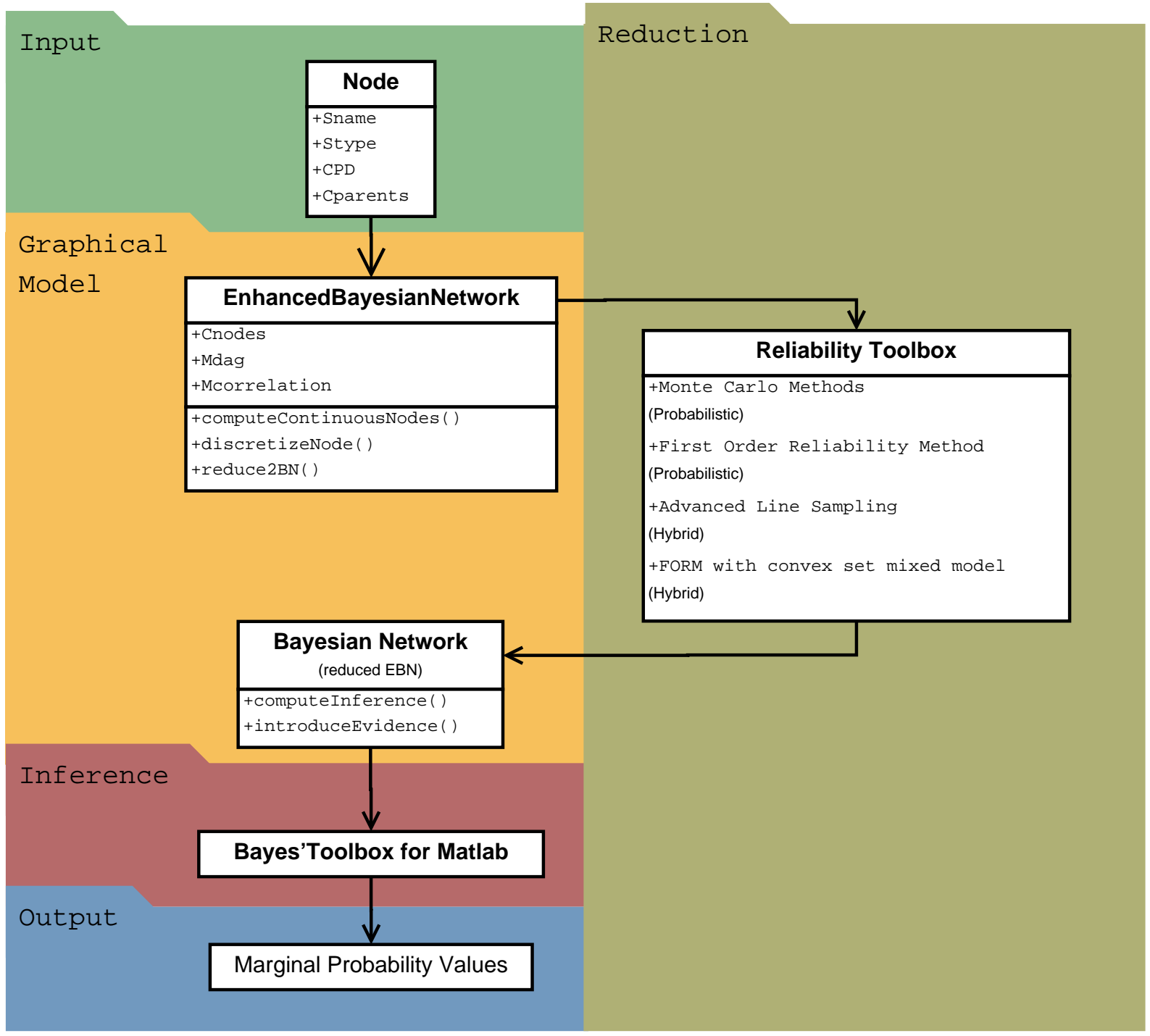

Figure 3: Simplified representation of the computational toolbox

\begin{tabular}{|c|c|c|c|}
\hline Property & Type & Required & Description \\
\hline Sname & String & Yes & Name of the node \\
\hline Cparents & Cell array of strings & Yes & Name of parent nodes \\
\hline Cchildren & Cell array of strings & No & Name of children nodes \\
\hline$C P D$ & Cell array & Yes & Conditional Probability Distribution \\
\hline Nsize & Integer & No & Size of the node \\
\hline Stype & String & Yes & $\begin{array}{l}\text { Type of node, i.e. discrete, probabilistic or } \\
\text { bounded }\end{array}$ \\
\hline Vbounds & Array of doubles & No & $\begin{array}{l}\text { Vector of bound values (in case of discrete or } \\
\text { discretized nodes) }\end{array}$ \\
\hline Evidence & Integer & No & $\begin{array}{l}\text { Value of the evidence eventually introduced in } \\
\text { the node }\end{array}$ \\
\hline
\end{tabular}

Table 1: Main properties of a Node object 
of bounded nodes it contains interval or a vector of interval variables; likewise, the CPD of probabilistic nodes can contains one or more random variables. In this latter case, OpenCossan offers a further grade of flexibility providing the options of defining random variables from a set of well-known distributions (specifying the moments), from data available or user defined functions.

In case of nodes children of at least one continuous node (i.e. bounded or probabilistic), the CPD is initially characterized by one or more scripts, representing the user defined models to be evaluated for the construction of the definitive CPD. These are expressed in the form of strings in which the variables involved are named according to the parent nodes.

\subsection{Enhanced Bayesian Network Class}

The object Enhanced Bayesian Network, which contains the model and the related information, is built introducing as input the node objects previously defined. Furthermore, a correlation matrix can be introduced: this way it is possible to consider the correlation among random variables or among interval variables (in the form of convex sets), whilst for other forms of correlation (e.g. between random and interval variables) it is necessary to represent it in the model itself (e.g. introducing a common parent). Apart form the correlation matrix and the node objects, other properties which characterize the EBN object can either be defined by the user or extrapolated from the input node objects. Overall, the main property of an EBN object are defined in Table 2.

\begin{tabular}{cccl}
\hline Property & Type & Required & Description \\
\hline Cnodes & Cell array & Yes & Cell array of Node objects \\
Cnames & Cell array of strings & No & Cell array of nodes names \\
Ctypes & Cell array of strings & No & Cell array of nodes type \\
Vsize & Cell array & Yes & Array of nodes size \\
Mdag & Matrix of integers & No & Directed acyclic graph adjacency matrix \\
Cevidence & Cell array & No & Cell array of evidence values \\
Cobserved & Cell array of strings & No & Cell array of observed nodes names \\
Nnodes & Integer & No & Number of nodes in the network \\
Mcorrelation & Matrix of doubles & No & Correlation matrix \\
\hline
\end{tabular}

Table 2: Main properties of a Node object

The methods of the class Enhanced Bayesian Network which directly modify the object can be divided in three categories according to as many tasks: methods dedicated to the modelling of the network, involved in the reduction procedure or in the inference computation. Not included in this classification is the method dedicated to the visualization of the graphical model, based on the use of the biograph toolbox for Matlab [9].

\subsubsection{Modelling}

The methods of this category have the capability of directly modify the topography of the network. Hence, this includes the removal of old nodes from the network, the inclusion of new nodes (e.g. those newly defined from the reduction procedure), the identification of barren nodes (namely those which do not receive any inference and have no children and so do not 
give any contribution towards the computation of the model) and the dicretization of continuous nodes. The latter, as specified in section 2.2, plays an essential role when a not-discrete variable needs to be included in the resulting BN. More generally, it is an important tool of modelling strategy (e.g. to preserve causal links in the reduced network). In order to minimize

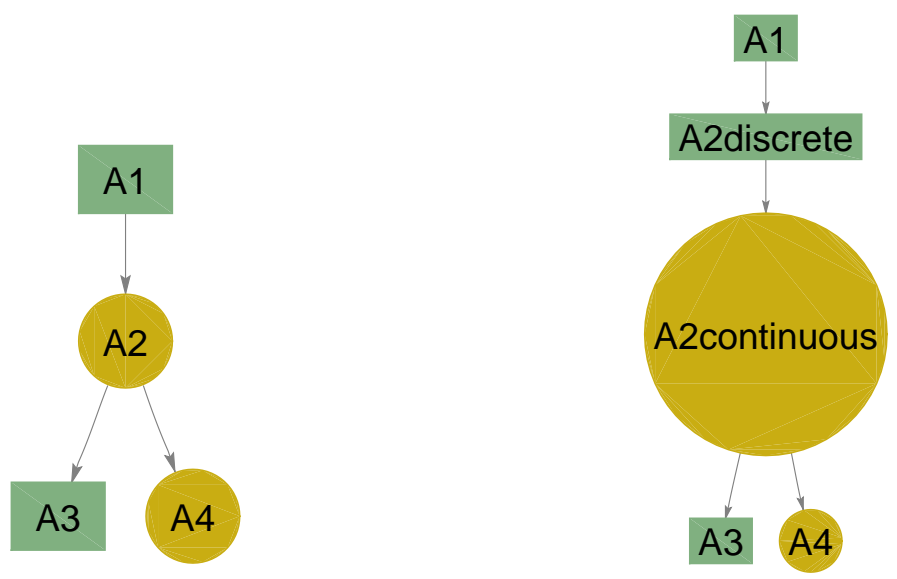

Figure 4: Graphical representation of the discretization procedure described

the impact of discretization on the quality of the information the procedure suggested by Straub and Kiureghiam [18] has been implemented computationally and extended to interval variables. Briefly, it consists in the substitution of the initial node with a discrete one (which inherit the parents of the initial node) associated to one or more continuous children (which inherit the children). The values of the discrete node CPT are equal to the cumulative probability values of the initial random variable sub-domains of discretization (in case of interval variables all the states are considered equally possible). Each of this state is then associated to a random variable of the continuous child which is built from the starting distribution on the sub-domain of discretization. This way neither the parents of the initial continuous nodes neither the children, are affected by the discretization procedure. Figure 4 summarizes graphically the procedure described.

\subsubsection{Reduction}

In case of discrete nodes children of at least one continuous node, the related CPT is computed through structural reliability methods. The nature and method of the reliability analysis to be carried out depends on the type of variables involved. If all the parents of the node to compute are modelled as random variables, probabilistic models are built for each combination of them. Hence, the mentioned models are subject to structural reliability analysis in order to compute the associated probability. This latter can be evaluated using FORM (default option) or traditional Monte Carlo approach (with a number of samples equal to $10^{5}$ if not specified otherwise). Likewise, in case of both intervals and random variables involved, the entries of the new CPT can be computed using either the Advanced Line Sampling method [3] or the generalized FORM [8] mentioned in section 2.2.1. In both the analysis the selection of one method is driven by the user choice of focusing on near-real time output rather than more accurate results or vice versa. In case of probabilistic nodes children of non-discrete variables, Monte Carlo methods with $10^{6}$ samples are used to collect data for the construction of the related random variables. 
Finally, in case of bounded nodes children of probabilistic and bounded parent nodes, a random search is carried out in the parents domain in order to find the bounds of the new interval variables.

The methods adopted for the computation of nodes children of at least one non-continuous variable, according to their type, are specified in Table 3. This procedure not only assigns to

\begin{tabular}{clll}
\hline Node Type & Parent Nodes & Method & New CPD \\
\hline Discrete & Random, Discrete & MC or FORM & CPT \\
Discrete & Random, & FORM with convex set models or & CPT \\
& Bounded,Discrete & Advanced Line Sampling \\
Bounded $*$ & $\begin{array}{l}\text { Random, } \\
\text { Bounded,Discrete }\end{array}$ & Random Search & Interval(s) \\
Random* & Random, Discrete & Monte Carlo & Random Variable(s) \\
\hline \multicolumn{2}{c}{$*$ to be removed after children nodes computation } &
\end{tabular}

Table 3: Methods for the computation of EBN nodes according to their type

the node under study the CDT computed through the reliability analysis but also ensures the elimination of the causal links between the node and its initial continuous parents. Indeed, the new discrete node inherits the discrete ancestors of its continuous parent nodes that, loosing this way all continuous children, can be finally removed from the network since barren. In the end, the elimination of all continuous nodes leads to the reduction of the initial Enhanced Bayesian Network to a traditional BN.

\subsubsection{Inference}

The inference computation on the network benefits from the capabilities of the Bayes'Toolbox for Matlab. Linking the OpenCossan implementation to the toolbox, the methods of this category allow to introduce evidence in the network and compute marginal probability values for the nodes of interest selecting the exact inference algorithms among those available (the default option being the Junction Tree algorithm).

\section{APPLICATION}

A simple application is introduced in this section. Firstly the case study is described together with the specifications of the input involved. The related EBN is then described and the results presented and analysed.

\subsection{Case Study and Model Implementation}

The case study proposed consists of a simple structure subject to horizontal and vertical loads. According to models available in literature [4, 18], three failure modes are considered and described by as many limit-state functions:

$$
\begin{array}{r}
g_{1}(\mathbf{x})=r_{1}+r_{2}+r_{4}+r_{5}-5_{h} \\
g_{2}(\mathbf{x})=r_{2}+2 r_{3}+r_{4}-5_{v} \\
g_{3}(\mathbf{x})=r_{1}+2 r_{3}+2 r_{4}+r_{5}-5_{h}-5_{v}
\end{array}
$$


where $R_{1}, R_{2}, R_{3}, R_{4}$ and $R_{5}$ represent the plastic moment capacities of the structure, whilst $H$ and $V$ are the horizontal and vertical loads respectively. The failure of the structure occurs when at least one of the failure modes is verified. In order to proof the Bayesian updating capabilities of EBN additional information in the form of measurements $\left(M_{4}, M_{5}\right)$ of the plastic moments $R_{4}, R_{5}$ is assumed to be available. The measurements are considered affected by an error $\varepsilon$ so that:

$$
\begin{aligned}
& M_{4}=R_{4}+\varepsilon \\
& M_{5}=R_{5}+\varepsilon
\end{aligned}
$$

Two cases have been analysed: in the first, all the variables are represented by random distributions (see Table 1). Conversely, in the second case the loads and the measurement error are represented by intervals, whose range is equal to 2 standard deviations of the distributions previously assigned to the same variables (see Table 2). In both examples the plastic moments capacities are considered equicorrelated with a linear correlation factor equal to 0.3.

\begin{tabular}{cccccc}
\hline Variable & Unit of Measurement & Distribution Type & Mean & STD & Correlation \\
\hline$R_{i}, i=1 \ldots 5$ & $k N \cdot m$ & Lognormal & 150 & 30 & 0.3 \\
$H$ & $k N$ & Gumbel & 50 & 20 & Independent \\
$V$ & $k N$ & Gamma & 60 & 12 & Independent \\
epsilon & & Normal & 0 & 15 & Independent \\
\hline
\end{tabular}

Table 4: Probabilistic models specification

The model related to the first case which takes into account only random variables, is shown in Fig.5a. The discrete nodes (rectangular shaped in the graph) FailureMode1, FailureMode2, FailureMode 3 refer to the occurrence of structural failure according to Eq. 7,8 and 9 respectively. The node $E$ is the only other discrete node in the initial network and represents the occurrence of the overall structural failure. The continuous nodes M4 and M5 refer to the measurements available and result from the true value of the related plastic capacities and the measurement error epsilon as shown in Eq.10. In order to take into account the evidence introduced on the nodes $M 4$ and $M 5$ these latter are automatically discretized together with their parents $R_{4}$ and $R_{5}$. On the contrary, the parent node epsilon is automatically eliminated from the model after the computation of the children nodes $M 4$ and $M 5$, since it plays no role in the inference computation neither in the structural connection of the evidence nodes to the overall network (see Fig.5b). Finally, as shown in Fig.5c, the continuous nodes originated from the discretization of M4 and M5 are eliminated since do not affect in any way the computation of the model (i.e. barren nodes).

\begin{tabular}{cccccc}
\hline Variable & Unit of Measurement & Mean & Lower Bound & Upper Bound & Correlation \\
\hline$H$ & $k N$ & 50 & 10 & 90 & Independent \\
$V$ & $k N$ & 60 & 20 & 100 & Independent \\
epsilon & & 0 & -30 & 30 & Independent \\
\hline
\end{tabular}

Table 5: Specifications of the interval variables of the model considering epistemic uncertainty

Similarly to the previous case, the EBN considering epistemic uncertain variables (Fig.6a) contains four discrete nodes initially. Nevertheless, the nodes $H$ and $V$ as well as the nodes 


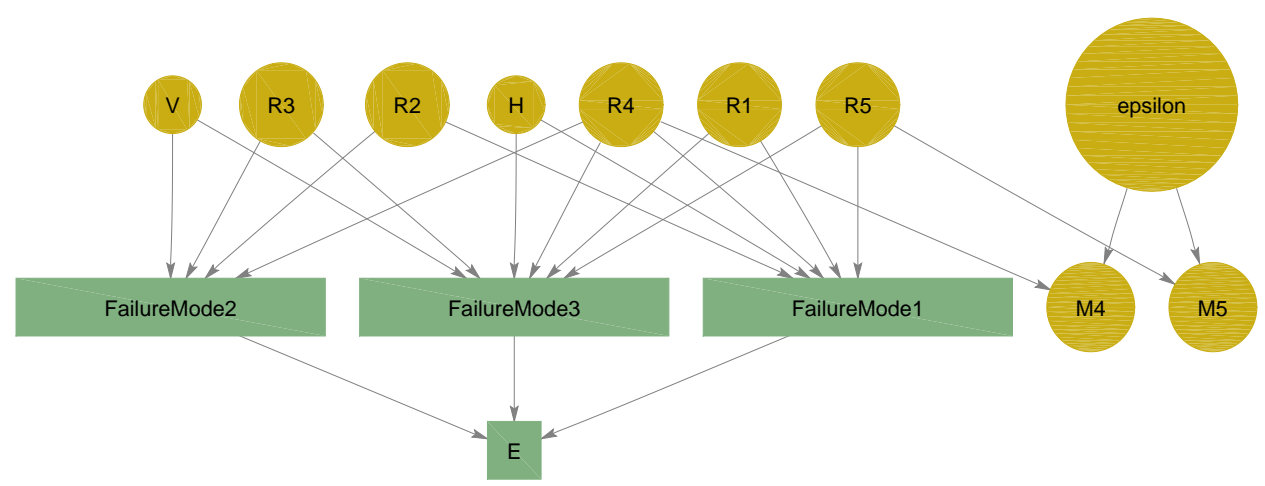

(a)

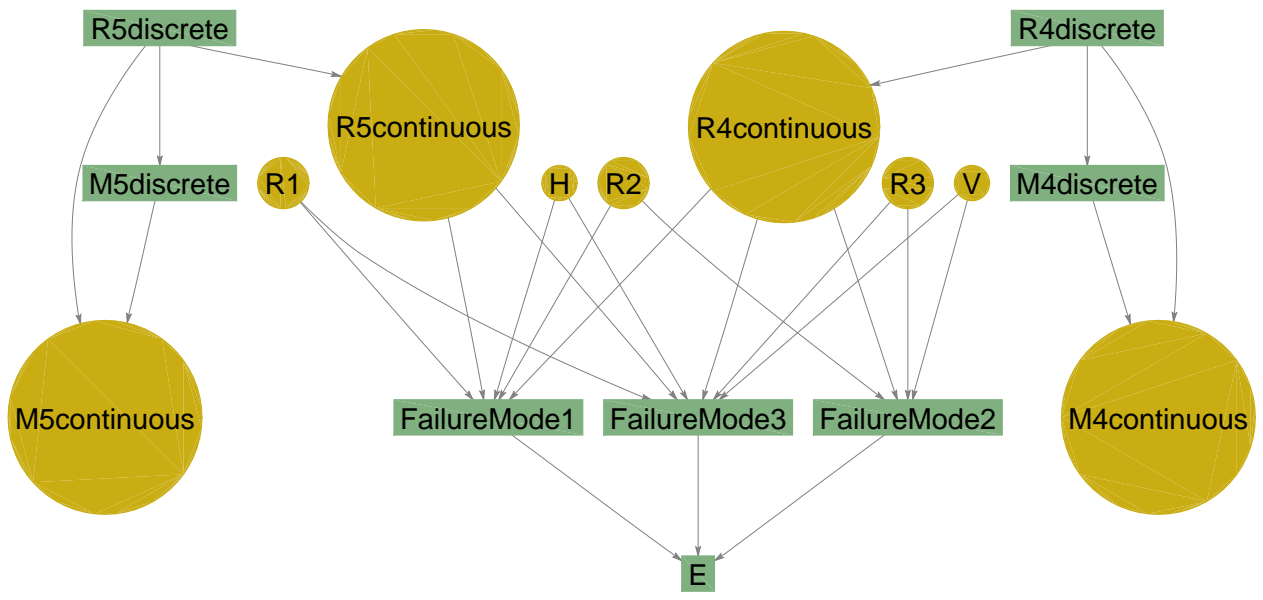

(b)

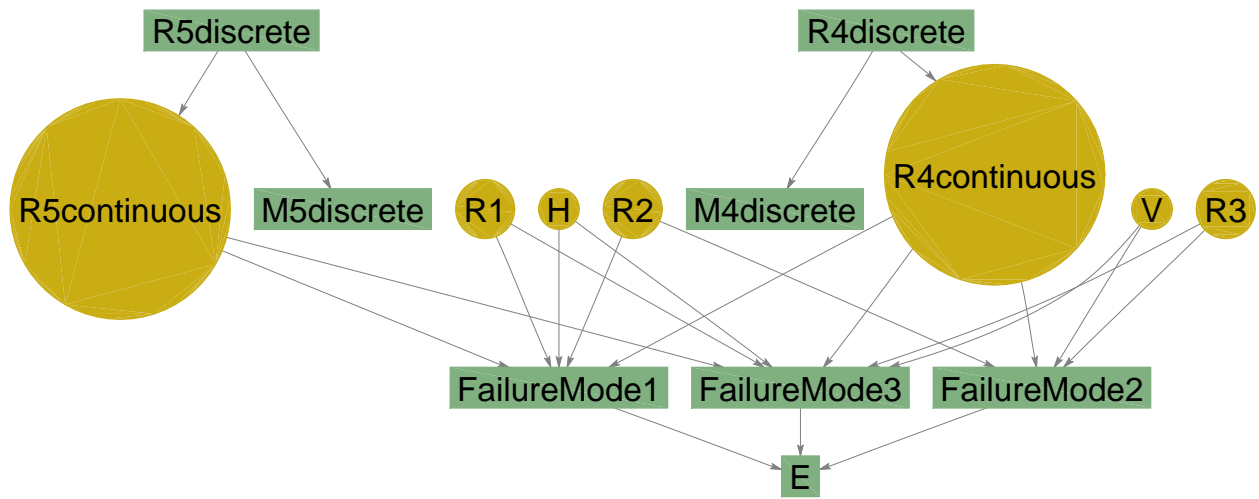

(c)

Figure 5: Evolution of the EBN model for the probabilistic analysis: (a) the initial model, (b) the model following the discretization of the variables of interest,(c) the model after the elimination of barren nodes

epsilon and its children are considered to be bounded variables (oval-shaped in Fig.6). Apart from this difference in the input, the procedure follows the same steps of the previous case, as shown by Fig. 6 . 


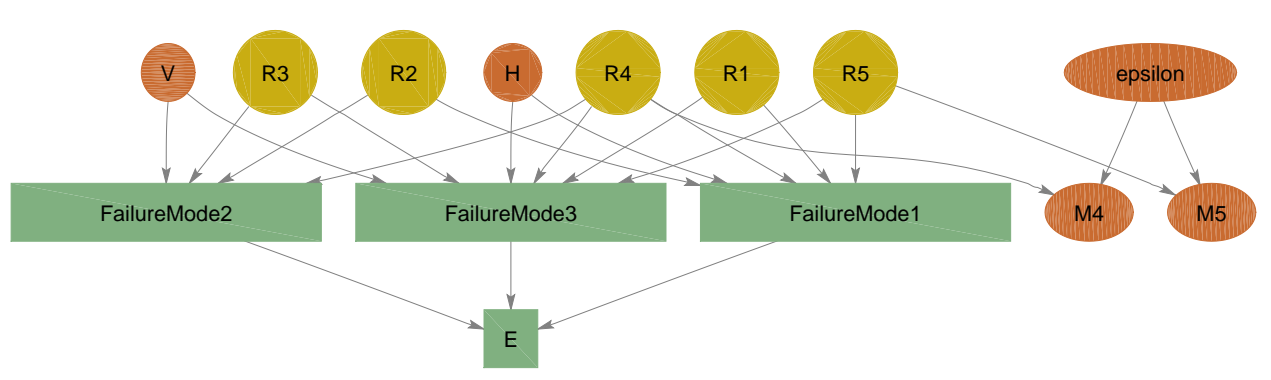

(a)
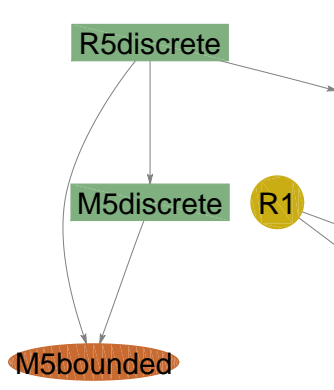
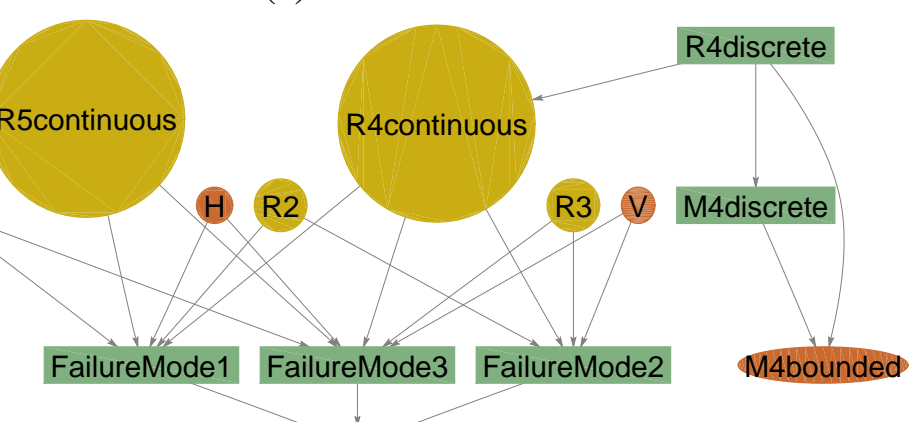

(b)

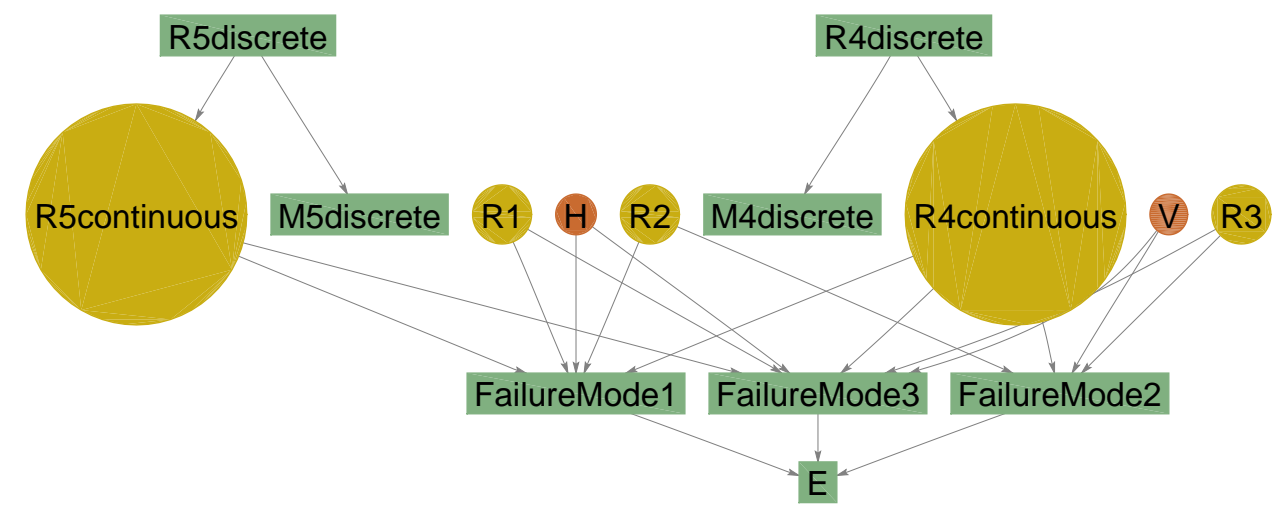

(c)

Figure 6: Evolution of the EBN model considering epistemic uncertainty: (a) the initial model, (b) the model following the discretization of the variables of interest,(c) the model after the elimination of barren nodes

\subsection{Results}

Both the initial models, with and without interval variables, result in the reduced $\mathrm{BN}$ of Fig.7. The numerical results have been computed using different structural reliability methods, considering 11 states for the discretization of nodes $R_{4}, R_{5}, M_{4}, M_{5}$. Table 6 shows the results of the analysis considering only probabilistic models for different values of evidence. The analysis has been carried out adopting Monte Carlo methods with $10^{6}$ samples and FORM. As the figures show, the probability of structural failure grows for lower values of plastic moments measurements, reaching a maximum of 0.211 . Thanks to the flexibility of BNs is also possible to analyse What-if scenarios. Table 7 shows the probability associated to each failure mode in case of occurrence of overall structural failure: the most probable mechanism of failure is the 


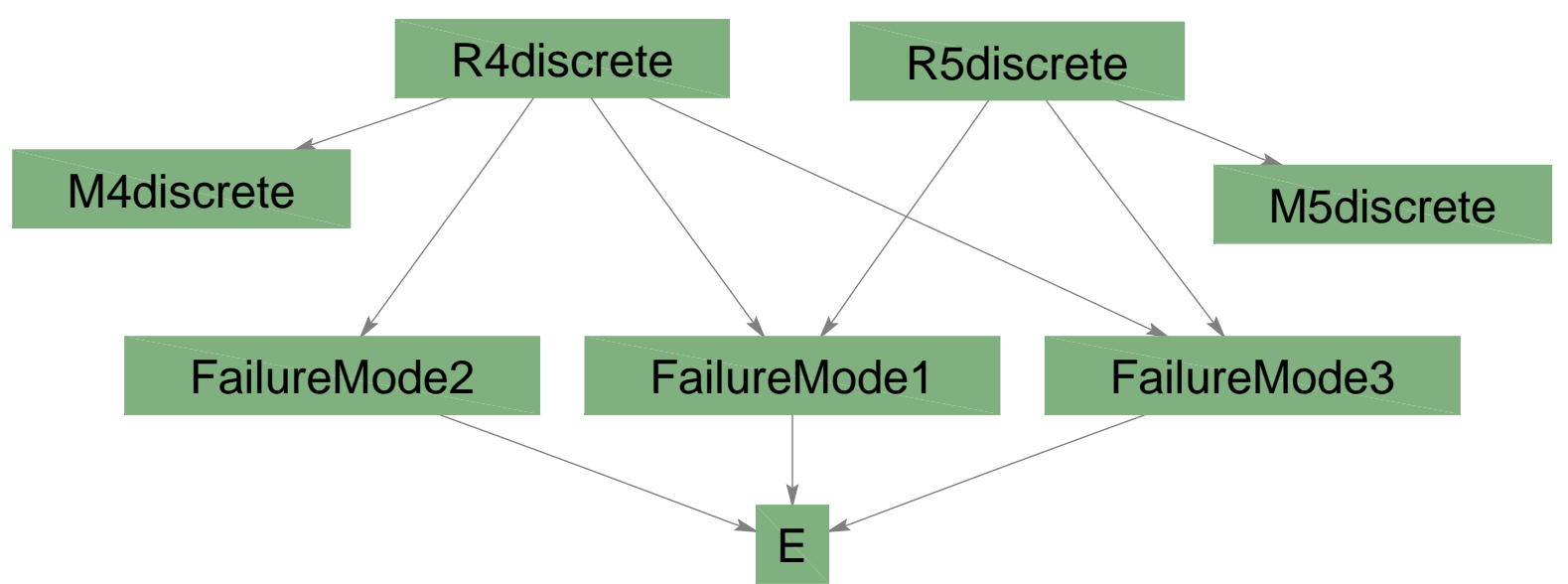

Figure 7: BN resulting from the reducing procedure applied on the two case studies analysed

\begin{tabular}{cccccc}
\hline Event & SRM & $\begin{array}{c}\text { No } \\
\text { Evidence }\end{array}$ & $\begin{array}{c}70>M_{4}>50 \\
90<M_{5}<110\end{array}$ & $\begin{array}{c}150>M_{4}>170 \\
90<M_{5}<110\end{array}$ & $150>M_{4}>170$ \\
\hline$E$ & MC & 0.099 & 0.211 & 0.116 & 0.0520 \\
$E$ & FORM & 0.100 & 0.202 & 0.116 & 0.0520 \\
\hline
\end{tabular}

Table 6: Results of the analysis considering only probabilistic model according to different values of evidence

third of the failure modes considered (described by Eq.9) for every combination of evidence values.

\begin{tabular}{|c|c|c|c|c|}
\hline If structural failure & No & $70>M_{4}>50$ & $150>M_{4}>170$ & $150>M_{4}>170$ \\
\hline occurred & Measurement & $90<M_{5}<110$ & $90<M_{5}<110$ & $190>M_{5}>210$ \\
\hline FailureMode1 & 0.093 & 0.107 & 0.095 & 0.085 \\
\hline FailureMode 2 & 0.008 & 0.015 & 0.004 & 0.009 \\
\hline FailureMode 3 & 0.909 & 0.902 & 0.912 & 0.910 \\
\hline
\end{tabular}

Table 7: Probability associated to each failure mode in case of failure of the structure

In Table 8 the results related to the analysis with uncertain bounded variables are presented for both the reliability methods adopted in the resolution of the model. As foreseeable, the

\begin{tabular}{cccccc}
\hline Event & SRM & $\begin{array}{c}\text { No } \\
\text { Evidence }\end{array}$ & $\begin{array}{c}70>M_{4}>50 \\
90<M_{5}<110\end{array}$ & $\begin{array}{c}150>M_{4}>170 \\
90<M_{5}<110\end{array}$ & $150>M_{4}>170$ \\
\hline$E$ & Ref. [8] & 0.491 & 0.582 & 0.499 & $M_{5}>210$ \\
\hline$E$ & Ref. [3] & 0.520 & 0.527 & 0.521 & 0.472 \\
\hline
\end{tabular}

Table 8: Results of the analysis considering epistemic uncertainty according to different values of evidence

introduction of intervals instead of precise probability distributions leads to significant higher values of the failure probability. Furthermore, the trend is much less sensible to fluctuations along with the increasing evidence values: this possibly indicates that the epistemic uncertainty 
introduced affects strongly the output values, making the influence of the evidence introduced almost negligible. In spite of this, it is still possible to observe a similar behaviour in the probability of failure which decreases with the growth of the measurements values introduced.

\section{CONCLUSIONS}

A computational tool for the reduction of Bayesian Networks enhanced with structural reliability methods is proposed. The algorithms allow to include in the initial network discrete, continuous as well as interval variables, providing a more accurate representation of the information available. Robust structural reliability methods are integrated in the framework in order to reduce the initial network to a traditional BN. This allows to adopt exact inference methods in the computation of the networks without limiting the analysis to only discrete variables. Two models of a same case study are proposed and analysed: the first relies only on probabilistic models (hence on the adoption of traditional structural reliability methods), the second one takes into account variables affected by epistemic uncertainty represented by intervals. The results of the two models are computed, compared and discussed.

In the case of models including epistemic uncertainty, only the upper bound of the probability computed through structural reliability methods is considered in the current implementation. On the one side, this is supposed to result in a conservative approach in case of failure events, on the other it leads to a loss of important information about the amount of uncertainty affecting the output and could lead to misleading estimations. Current efforts are dealing with extension of the methodology to allow the estimation of the probability bounds hence to represent the propagation of epistemic uncertainty from the input to the output of the network.

\section{REFERENCES}

[1] Michael Beer, Scott Ferson, and Vladik Kreinovich. Imprecise probabilities in engineering analyses. Mechanical Systems and Signal Processing, 2013.

[2] Yakov Ben-Haim. A non-probabilistic concept of reliability. Structural safety, 14(4):227245, 1994.

[3] Marco de Angelis, Edoardo Patelli, and Michael Beer. Advanced line sampling for efficient robust reliability analysis. Structural Safety, 52:170-182, 2015.

[4] Armen Der Kiureghian et al. First-and second-order reliability methods. Engineering design reliability handbook, pages 14-1, 2005.

[5] John Michael Hammersley and David Christopher Handscomb. Monte carlo methods, volume 1. Methuen London, 1964.

[6] Abraham M Hasofer and Niels C Lind. Exact and invariant second-moment code format. Journal of the Engineering Mechanics division, 100(1):111-121, 1974.

[7] C Jiang, X Han, GY Lu, J Liu, Z Zhang, and YC Bai. Correlation analysis of nonprobabilistic convex model and corresponding structural reliability technique. Computer Methods in Applied Mechanics and Engineering, 200(33):2528-2546, 2011.

[8] Yangjun Luo, Zhan Kang, and Alex Li. Structural reliability assessment based on probability and convex set mixed model. Computers \& Structures, 87(21):1408-1415, 2009. 
[9] MathWorks. Bioinformatics toolbox, user's guide, r2014b version, October 2014. data available from: http://www.mathworks.co.uk/

[10] Ramon Moore and Weldon Lodwick. Interval analysis and fuzzy set theory. Fuzzy Sets and Systems, 135(1):5-9, 2003.

[11] Kevin Murphy. Software for graphical models: A review. International Society for Bayesian Analysis Bulletin, 14(4):13-15, 2007.

[12] Kevin P. Murphy. The Bayes Net Toolbox for Matlab. Computing science and statistics, 33(2):1024-1034, 2001.

[13] ER Neapolitan and Scott Morris. Probabilistic modelling with bayesian networks. The SAGE handbook of quantitative methodology for the social sciences, pages 371-390, 2004.

[14] Edoardo Patelli, H Murat Panayirci, Matteo Broggi, Barbara Goller, Pierre Beaurepaire, Helmut J Pradlwarter, and Gerhart I Schuëller. General purpose software for efficient uncertainty management of large finite element models. Finite Elements in Analysis and Design, 51:31-48, 2012.

[15] Judea Pearl and Stuart Russell. Bayesian networks, November 2000. https:// escholarship.org/uc/item/53n4f34m

[16] Rüdiger Rackwitz. Reliability analysisa review and some perspectives. Structural safety, 23(4):365-395, 2001.

[17] Stuart Jonathan Russell and Peter Norvig. Artificial intelligence : a modern approach. Upper Saddle River, N.J. ; Pearson Education, 2010., 2010.

[18] D. Straub and Der Kiureghian. Bayesian network enhanced with structural reliability methods: methodology. Journal of engineering mechanics, 136(10):12481258, 2010.

[19] Silvia Tolo, Edoardo Patelli, and Michael Beer. Bayesian network approach for risk assessment of a spent nuclear fuel pond. In Vulnerability, Uncertainty, and Risk Quantification, Mitigation, and Management, pages 598-607. ASCE.

[20] Fulvio Tonon. Using random set theory to propagate epistemic uncertainty through a mechanical system. Reliability Engineering \& System Safety, 85(1):169-181, 2004.

[21] Philippe Weber, Gabriela Medina-Oliva, Christophe Simon, and Benoît Iung. Overview on Bayesian networks applications for dependability, risk analysis and maintenance areas. Engineering Applications of Artificial Intelligence, 25(4):671-682, 2012.

[22] Lotfi A Zadeh. Fuzzy sets. Information and control, 8(3):338-353, 1965. 